УДК 681.7.068/.069

\author{
В.А. Акулов ${ }^{1,2}$, А.А. Власов² \\ ${ }^{1}$ ООО «Инверсия-Файбер», Новосибирск, Россия \\ ${ }^{2}$ Институт автоматики и электрометрии СО РАН, Новосибирск, Россия
}

\author{
УЛЬТРАФИОЛЕТОВЫЙ ЛАЗЕР \\ НА ОСНОВЕ ОДНОЧАСТОТНОГО ВОЛОКОННОГО \\ РОС-ЛАЗЕРА С ДЛИНОЙ ВОЛНЫ 1030 НМ
}

\begin{abstract}
Разработан ультрафиолетовый лазер на основе одночастотного волоконного лазера с длиной волны генерации 1030 нм. С помощью периодически ориентированного кристалла PPSLT получено излучение второй гармоники 515 нм, которое преобразовано во внешнем резонаторе в 4-ю гармонику 257,5 нм в кристалле ВВО. В качестве задающего лазера был использован иттербиевый лазер с распределенной обратной связью (РОС-лазер), который затем был усилен в нескольких каскадах волоконных усилителей до мощности 10 Вт. На длине волны 515 нм при этом мощность достигла 1,5 Вт. Данного уровня было достаточно, чтобы получить 100 мВт 4-й гармоники в УФ-диапазоне. На базе полученных результатов разработан относительно компактный и энергоэффективный источник УФ-излучения, не требующий водяного охлаждения.
\end{abstract}

Ключевые слова: волоконный лазер с распределённой обратной связью, иттербиевый, ультрафиолетовый лазер, четвертая гармоника, внешний резонатор.

\author{
V.A. Akulov ${ }^{1,2}$, A.A. Vlasov ${ }^{2}$ \\ ${ }^{1}$ Inversion Fiber Co. Ltd., Novosibirsk, Russian Federation \\ ${ }^{2}$ Institute of Automation and Electrometry, Novosibirsk, Russian Federation

\section{ULTRAVIOLET LASER BASED ON SINGLE FREQUENCY DFB FIBER LASER WITH 1030 NM WAVELENGTH}

There was developed the UV laser based on single frequency fiber laser with wavelength $1030 \mathrm{~nm}$. The second harmonic $515 \mathrm{~nm}$ was obtained with PPSLT crystal, which was converted to the fourth harmonic $257.5 \mathrm{~nm}$ in external cavity. An ytterbium doped distributed feedback fiber laser (DFB fiber laser) was used as a seed laser. The radiation of the DFB laser was amplified in few fiber amplifiers to $10 \mathrm{~W}$. With this power there was achieved $1.5 \mathrm{~W}$ at $515 \mathrm{~nm}$. This power level was enough to obtain $100 \mathrm{~mW}$ in UV region of spectra. On the basis of these results the compact and energy efficient UV source was developed, which does not require water cooling. cavity.

Keywords: distributed feedback fiber laser, Yb-doped, UV laser, fourth harmonic, external 


\section{Введение}

Создание волоконных брэгговских решеток (ВБР) является неотъемлемой частью современной волоконной оптики и волоконных лазеров. Процесс записи решёток эффективен при использовании ультрафиолетового (УФ) излучения [1]. Спектр поглощения германосиликатного волоконного световода имеет пик с центром на длине волны $\sim 240$ нм (рис. 1). В качестве источников ультрафиолетового излучения используются разные типы лазеров. В настоящее время наилучшими

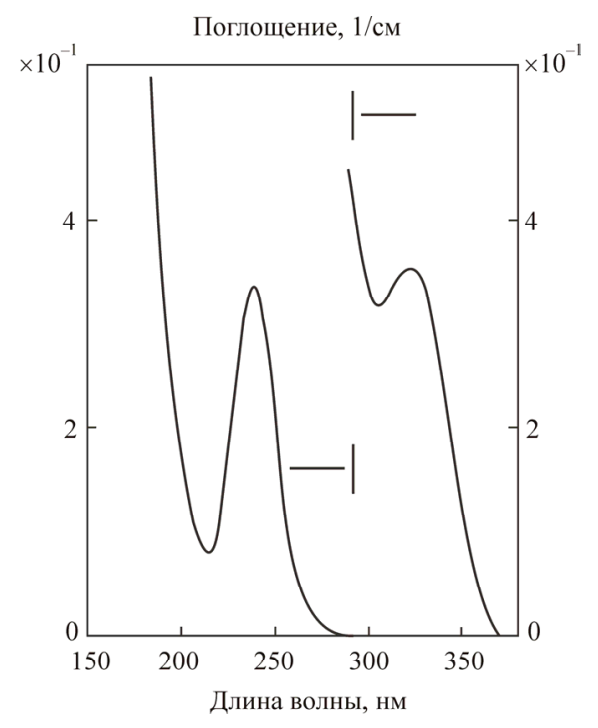

Рис. 1. Спектр поглощения германосиликатного волоконного световода характеристиками (длина когерентности, стабильность, качество пучка) для записи ВБР в германосиликатных волоконных световодах обладает излучение с длиной волны 244 нм, получаемое при удвоении частоты непрерывного аргонового лазера, а наиболее распространенными и относительно дешёвыми являются импульсные эксимерные лазеры с длиной волны 248 нм. Однако аргоновый лазер представляет собой сложную систему, состоящую из вакуумированной лазерной трубки, источника питания и насоса, необходимого для циркуляции охлаждающей жидкости в лазерной трубке. Такая система требует участия высококвалифицированного специалиста для управления и обслуживания. Основные эксплуатационные расходы связаны с оплатой электроэнергии и воды и необходимостью регулярного ремонта/замены вакуумной газоразрядной трубки. Эксимерный лазер более эффективен, чем аргоновый, по энергетике, но с гораздо худшим качеством пучка и импульсным режимом генерации, что ограничивает возможности записи ВБР только самыми простыми вариантами решёток в схеме с фазовой маской.

В качестве альтернативы для записи ВБР может служить предлагаемый иттербиевый волоконный лазер (ИВЛ) с генерацией 4-й гармоники в кристалле ВВО. При длине волны ИВЛ 1030 нм длина волны 
4-й гармоники составит 257,5 нм, для которой фоточувствительность германосиликатного волоконного световода примерно в 5-6 раз меньше, чем для 244 нм. Соответственно время экспозиции ВБР увеличивается во столько же раз при одинаковом уровне мощности аргонового и волоконного лазеров. К преимуществам ИВЛ по сравнению с аргоновым лазером можно отнести следующие характеристики: гораздо меньшие размеры и масса, более простое управление и обслуживание, низкие энергозатраты (потребление меньше примерно в 250 раз), отсутствие водяного охлаждения, относительно низкая стоимость как самого лазера, так и расходов на его эксплуатацию. Основные эксплуатационные расходы связаны с заменой нелинейного кристалла ВВО.

\section{Эксперименты и результаты}

Принципиальная оптическая схема лазера представляет из себя последовательность «задающий генератор - усилитель - генератор второй гармоники - генератор четвертой гармоники».

Задающим генератором является волоконный иттербиевый POC-лазер, записанный с помощью УФ аргонового лазера и фазовой маски в иттербиевом волокне с сохранением поляризации [2]. Выходная мощность задающего генератора составила $20 \mathrm{мB}$ при мощности накачки 500 мВт на 976 нм. Измеренная длина волны одночастотной генерации линейно поляризованного излучения при этом составила 1030,2 нм, ширина линии 100 кГц. Далее излучение было усилено аналогично [3] в нескольких каскадах волоконных усилителей до уровня мощности 10 Вт. Выходной каскад усилителя был построен на базе активного волокна с сохранением поляризации Nufern PLMA-YDF-10/125-M. Данное волокно на длине волны 1030 нм имеет параметр $V=2,516$. Известно, что для поддержания одной поперечной моды в волоконном световоде значение параметра $V$ не должно превышать 2,405 [4]. Измерение параметров пучка при выходной мощности 10 Вт показало, что параметр качества пучка $M^{2} \leq 1,05$ (рис. 2) характерен для профиля поперечной моды $\mathrm{TEM}_{00}$. Также были измерены шумовые характеристики лазерного излучения. На рис. 3 представлен спектр относительного шума интенсивности, свидетельствующий о том, что после усиления спектральная плотность шума остается достаточно низкой в широком диапазоне частот. 


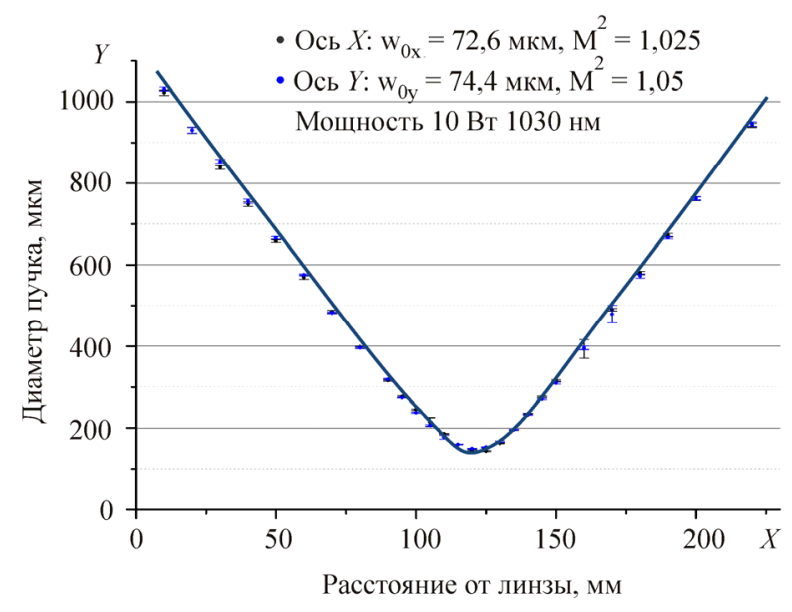

Рис. 2. Характеристики сфокусированного пучка одночастотного лазера с длиной волны 1030 нм и выходной мощностью 10 Вт

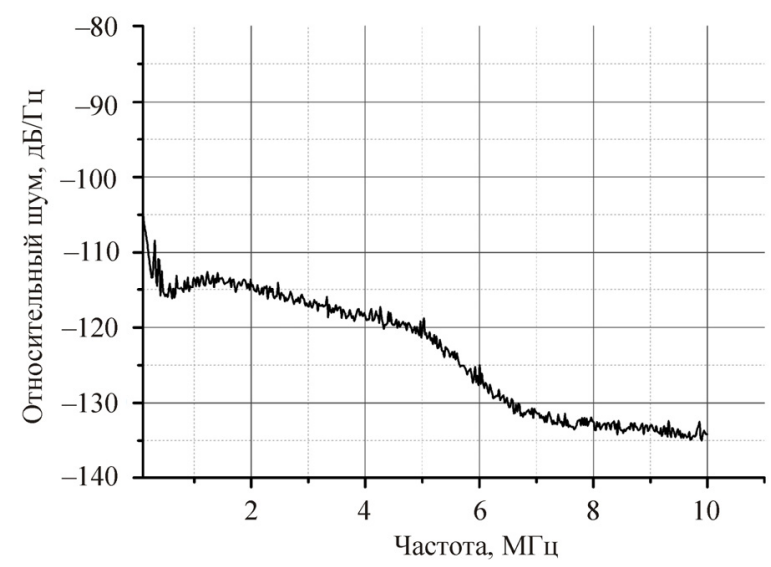

Рис. 3. Спектр относительного шума интенсивности одночастотного волоконного лазера на длине волны 1030 нм мощностью 10 ВТ

Для генерации второй гармоники (ГВГ) был использован периодически ориентированный кристалл PPSLT длиной 30 мм. В отличие от кристаллов PPLN для PPSLT характерна меньшая эффективность преобразования, однако эффект фоторефракции, приводящий к потемнению под действием видимого излучения и даже разрушению кристалла, имеет значительно больший порог для PPSLT [5, 6]. Согласно теории Бойда и Кленмана для ГВГ в сфокусированном гауссовом пучке [7] диаметр перетяжки пучка для оптимального преобразования во 
2-ю гармонику в 30 мм кристалле PPSLT составляет 56 мкм. В нашем случае диаметр перетяжки составил 60 мкм. При этом из 10 Вт основного излучения на 1030 нм было получено 1,5 Вт вертикально поляризованного излучения 2-й гармоники на длине волны 515 нм.

Дальнейшее преобразование в УФ-диапазон осуществлялось с помощью четырехзеркального внешнего резонатора (рис. 4), аналогично удвоению частоты одночастотного аргонового лазера [8]. Вертикальная поляризация излучения генератора 2-й гармоники 1 поворачивается на $90^{\circ}$ с помощью полуволновой пластинки 2. С помощью поворотных зеркал 3 и согласующей линзы 4 излучение заводится во внешний резонатор 5. Резонатор состоит из следующих элементов: $5.1,5.2,5.3$ и 5.5 - зеркала резонатора; 5.4 - нелинейный кристалл ВВО; 6 - фотодиод для стабилизации резонатора. С помощью выходного спектрального фильтра 8 УФ-излучение отделяется от остаточного излучения 515 нм. Часть УФ-излучения подаётся на измерительный фотодиод 7. Стабилизация резонатора осуществляется с помощью электронной системы автоматической подстройки частоты. В системе стабилизации задействованы зеркало 5.2 (медленная петля обратной связи), зеркало 5.3 (быстрая петля обратной связи) и фотодиод 6 для регистрации сигнала излучения 515 нм. Данная конфигурация позволила достичь мощности 100 мВт на длине волны 257,5 нм при мощности излучения 1,5 Вт на 515 нм.

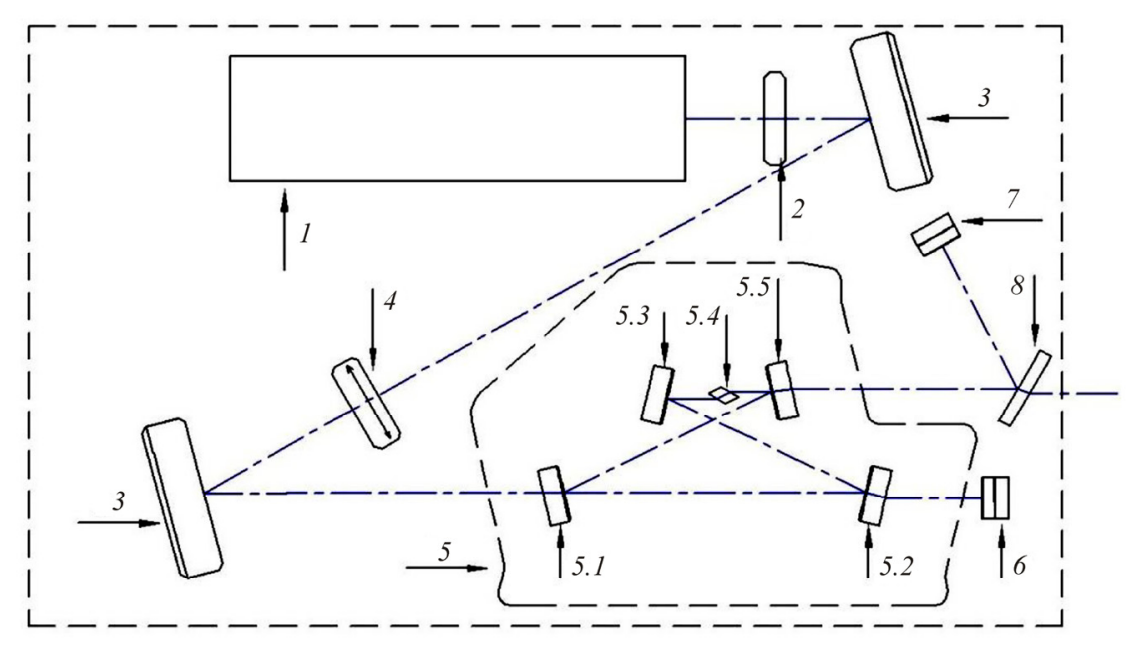

Рис. 4. Схема заведения излучения 515 нм во внешний резонатор для генерации 4-й гармоники 257,5 нм 
На базе полученных результатов был реализован приборный вариант волоконной лазерной системы УФ-диапазона излучения. На рис. 5 представлен внешний вид системы, на рис. 6 - габаритный чертеж. Лазерная система конструктивно состоит из трех функциональных модулей:

1) лазерного источника, генерирующего одночастотное излучение на длине волны 515 нм с выходной мощностью до 1,5 Вт. Габаритные размеры $457 \times 140 \times 395 \mathrm{mм}^{3}$;

2) оптического модуля, содержащего комплекс оптических компонентов и механических узлов, предназначенных для преобразования излучения 515 нм во 2-ю гармонику с использованием принципа удвоения частоты во внешнем резонаторе. Габаритные размеры $300 \times 105 \times 551 \mathrm{MM}^{3}$;

3) блока автоматической подстройки частоты (Блок АПЧ), предназначенного для управления и стабилизации блока внешнего резонатора, установленного в оптическом модуле. Габаритные размеры $457 \times 75 \times 395 \mathrm{mм}^{3}$.

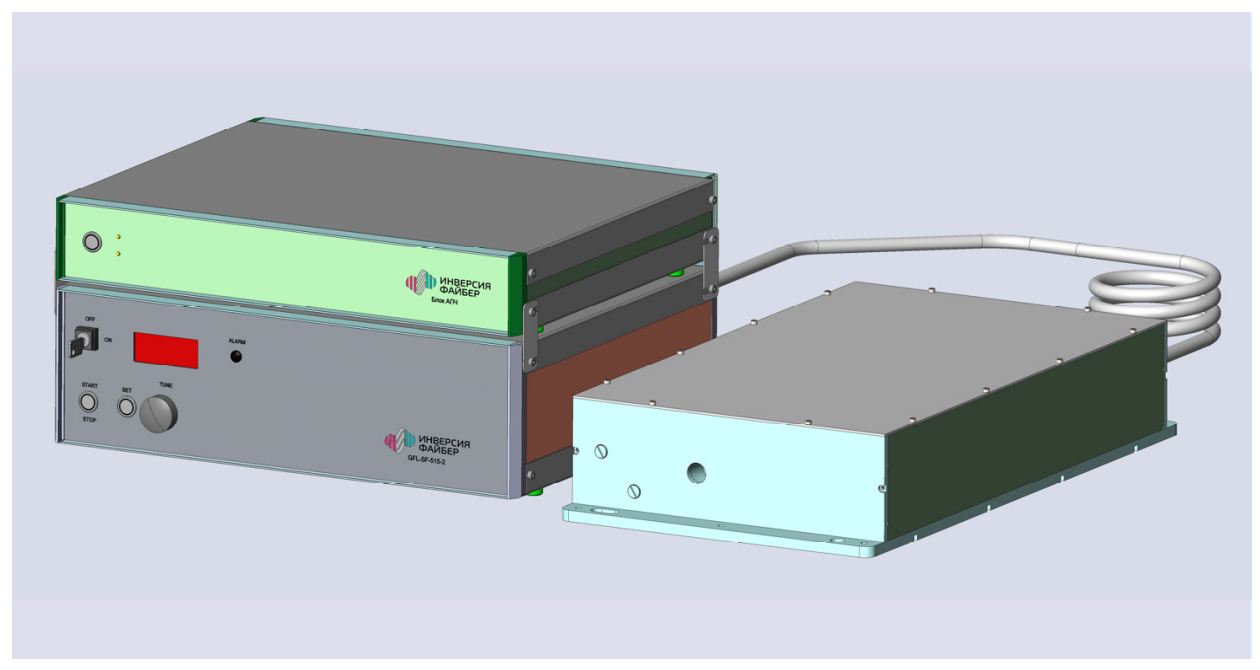

Рис. 5. Внешний вид волоконной лазерной системы УФ-диапазона

Суммарная мощность потребления от сети данной системы не превышает 500 Вт. 

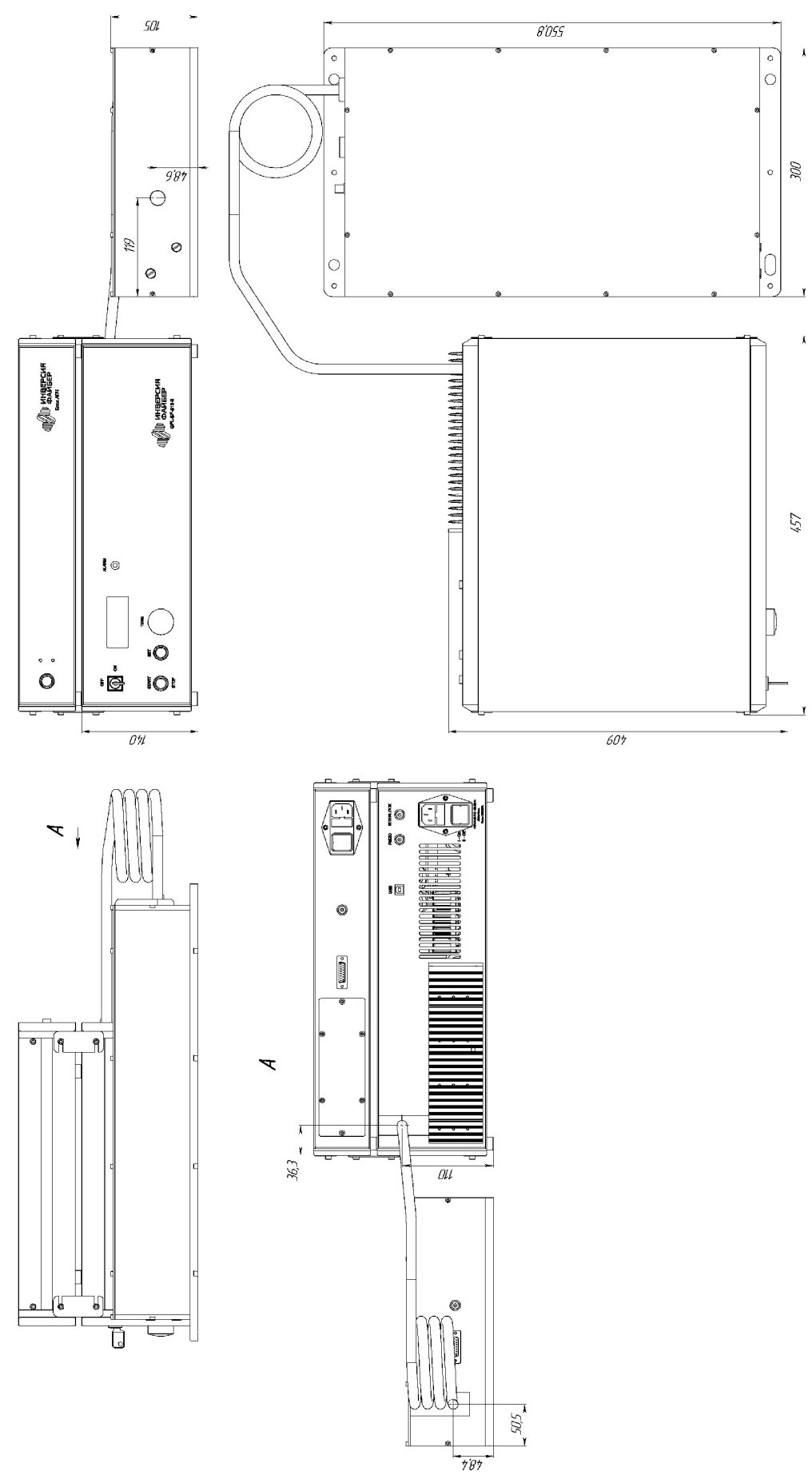

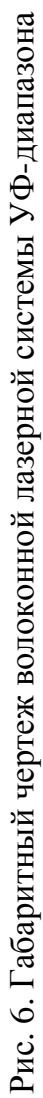




\section{Заключение}

В результате экспериментальной работы создан одночастотный волоконный лазерный источник линейно поляризованного излучения с длиной волны 1030 нм и мощностью 10 Вт. Преобразование во 2-ю гармонику позволило получить излучение видимого диапазона на длине волны 515 нм мощностью 1,5 Вт. С помощью внешнего резонатора удалось достичь мощности 100 мВт излучения 4-й гармоники в УФ-диапазоне на длине волны 257,5 нм.

На основе данных результатов создана довольно компактная и энергоэффективная лазерная система УФ-диапазона, которая может найти применение в области записи ВБР. Более того, подобные системы имеют огромный потенциал в области фотолитографии, где они могут заменить использующиеся на данный момент аргоновые лазеры УФ-диапазона.

Часть работы (исследование РОС-лазера) выполнена в рамках темы госзадания ИАиЭ СО РАН (№ 0319-2018-0004).

\section{Список литературы}

1. Lam D.K.W., Garside B.K. Characterization of single-mode optical fiber filters // Appl. Opt. - 1981. - Vol. 20. - P. 440-445.

2. Vlasov A.A., Churin D.E., Babin S.A. The features of characterization of the fiber Bragg gratings with phase shift for the distributed-feedback lasers written in the polarization-maintaining Ytterbium-doped fiber // Laser Physics - 2010. - Vol. 20, No. 12. - P. 2045-2049.

3. Иттербиевый волоконный лазер с распределенной обратной связью с низким уровнем частотных шумов / М.А. Никулин, С.А. Бабин, А.К. Дмитриев, А.С. Дычков, С.И. Каблуков, А.А. Луговой, Ю.Я. Печерский // Квант. Электроника - 2009. - Т. 39, № 10. - С. 906-910.

4. Snyder A.W., Love J.D. Optical Waveguide Theory // Chapman and Hall. - London, 1983.

5. Tovstonog S.V., Kurimura S., Kitamura K. High power continuous-wave green light generation by quasiphase matching in $\mathrm{Mg}$ stoichiometric lithium tantalite // Appl. Phys. Lett. - 2007. - Vol. 90. - P. 051115-1051115-3.

6. Frequency-doubling of a CW fiber laser using PPKTP, PPMgSLT, and PPMgLN / F.J. Kontur, I. Dajani, Yalin Lu, R.J. Knize // Opt. Express. 2007. - Vol. 15, No. 20. - P. 12882-12889. 
7. Boyd G.D., Kleinman D.A. Parametric interaction of focused Gaussian light beams // J. Appl. Phys. - 1968. - Vol. 39. - P. 3597-3639.

8. Babin S.A., Kablukov S.I., Vlasov A.A. Frequency doubling in the enhancement cavity with single focusing mirror // Proceedings SPIE / ed. V.I. Ustugov. - Bellingham, Washington: SPIE, 2004. - Vol. 5478. p. $165-172$.

\section{References}

1. Lam D.K.W., Garside B.K. Characterization of single-mode optical fiber filters. Appl. Opt., 1981, vol. 20, pp. 440-445.

2. Vlasov A.A., Churin D.E., Babin S.A. The features of characterization of the fiber Bragg gratings with phase shift for the distributed-feedback lasers written in the polarization-maintaining Ytterbium-doped fiber. Laser Physics, 2010, vol. 20, no. 12, pp. 2045-2049.

3. Nikulin M.A., Babin S.A., Dmitriev A.K., Dychkov A.S., Kablukov S.I., Lugovoy A.A., Pecherskiy Yu.Ya. Itterbievyy volokonnyy lazer s raspredelennoy obratnoy svyazyu s nizkim urovnem chastotnykh shumov [Ytterbium fiber laser using distributed feedback with low-frequency noise]. Kvant. Elektronika, 2009, vol. 39, no. 10, pp. 906-910.

4. Snyder A.W., Love J.D. Optical waveguide theory. London, Chapman and Hall, 1983.

5. Tovstonog S.V., Kurimura S., Kitamura K. High power continuouswave green light generation by quasiphase matching in $\mathrm{Mg}$ stoichiometric lithium tantalite. Appl. Phys. Lett., 2007, vol. 90, pp. 051115-1-051115-3.

6. Kontur F.J., Dajani I., Lu Yalin, Knize R.J. Frequency-doubling of a CW fiber laser using PPKTP, PPMgSLT, and PPMgLN. Opt. Express., 2007, vol. 15, no. 20, pp. 12882-12889.

7. Boyd G.D., Kleinman D.A. Parametric interaction of focused Gaussian light beams. J. Appl. Phys., 1968, vol. 39, pp. 3597-3639.

8. Babin S.A., Kablukov S.I., Vlasov A.A. Frequency doubling in the enhancement cavity with single focusing mirror. Proceedings SPIE. Ed. V.I. Ustugov. Bellingham, Washington: SPIE, 2004, vol. 5478, pp. 165-172.

Получено 17.05.2018 